\title{
Recommendations from the EGAPP Working Group: genetic testing strategies in newly diagnosed individuals with colorectal cancer aimed at reducing morbidity and mortality from Lynch syndrome in relatives
}

\author{
Evaluation of Genomic Applications in Practice and Prevention (EGAPP) Working Group*
}

\begin{abstract}
Disclaimer: This recommendation statement is a product of the independent EGAPP Working Group. Although the Centers for Disease Control and Prevention (CDC) provides support to the EGAPP Working Group, including staff support in the preparation of this document, recommendations made by the EGAPP Working Group should not be construed as official positions of the CDC or the U.S. Department of Health and Human Services.
\end{abstract}

\begin{abstract}
Summary of Recommendations: The Evaluation of Genomic Applications in Practice and Prevention (EGAPP) Working Group found sufficient evidence to recommend offering genetic testing for Lynch syndrome to individuals with newly diagnosed colorectal cancer to reduce morbidity and mortality in relatives. We found insufficient evidence to recommend a specific genetic testing strategy among the several examined.

Rationale: Genetic testing to detect Lynch syndrome in individuals with newly diagnosed colorectal cancer (CRC) is proposed as a strategy to reduce CRC morbidity and mortality in their relatives (see Clinical Considerations section for definition of Lynch syndrome). The EGAPP Working Group (EWG) constructed a chain of evidence that linked genetic testing for Lynch syndrome in patients with newly diagnosed $\mathrm{CRC}$ with improved health outcomes in their relatives. We found that assessing patients who have newly diagnosed CRC with a series of genetic tests could lead to the identification of Lynch syndrome. Relatives of patients with Lynch syndrome could then be offered genetic testing, and, where indicated, colorectal, and possibly endometrial, cancer surveillance, with the expectation of improved health outcome. The EWG concluded that there is moderate certainty that such a testing strategy would provide moderate population benefit. Analytic Validity:
\end{abstract}

\footnotetext{
*EGAPP Working Group: Chair: Alfred O. Berg, MD, MPH (University of Washington), Members: Katrina Armstrong, MD, MSCE (University of Pennsylvania School of Medicine); Jeffrey Botkin, MD, MPH (University of Utah); Ned Calonge, MD, MPH (Colorado Department of Public Health and Environment); James Haddow, MD (The Warren Alpert Medical School of Brown University); Maxine Hayes, MD, MPH (Washington State Department of Health); Celia Kaye, MD, PhD (University of Colorado School of Medicine); Kathryn A. Phillips, PhD (University of California, San Francisco); Margaret Piper, PhD, MPH (Blue Cross/Blue Shield Association Technology Evaluation Center); Carolyn Sue Richards, PhD, FACMG (Oregon Health \& Science University); Joan A. Scott, MS, CGC (Johns Hopkins University); Ora L. Strickland, PhD, DSc (Hon.), RN, FAAN (Emory University); Steven Teutsch, MD, MPH (Merck \& Co.).

E-mail: egappinfo@egappreviews.org.
}

Disclosure: Steven Teutsch is an employee, option and stock holder in Merck \& Co., Inc.

Submitted for publication August 13, 2008.

Accepted for publication September 29, 2008

DOI: $10.1097 /$ GIM.0b013e31818fa2ff
The EWG found adequate evidence to conclude that the analytic sensitivity and specificity for preliminary and diagnostic tests were high. Clinical Validity: After accounting for the specific technologies and numbers of markers used, the EWG found at least adequate evidence to describe the clinical sensitivity and specificity for three preliminary tests, and for four selected testing strategies. These measures of clinical validity varied with each test and each strategy (see Clinical Considerations section). Clinical Utility: The EWG found adequate evidence for testing uptake rates, adherence to recommended surveillance activities, number of relatives approachable, harms associated with additional follow-up, and effectiveness of routine colonoscopy. This chain of evidence supported the use of genetic testing strategies to reduce morbidity/mortality in relatives with Lynch syndrome. Several genetic testing strategies were potentially effective, but none was clearly superior. The evidence for or against effectiveness of identifying mismatch repair (MMR) gene mutations in reducing endometrial cancer morbidity or mortality was inadequate. Contextual Issues: CRC is a common disease responsible for an estimated 52,000 deaths in the United States in 2007 . In about $3 \%$ of newly diagnosed CRC, the underlying cause is a mutation in a MMR gene (Lynch syndrome) that can be reliably identified with existing laboratory tests. Relatives inheriting the mutation have a high (about $45 \%$ by age 70 ) risk of developing CRC. Evidence suggests these relatives will often accept testing and increased surveillance. Genet Med 2009:11(1):35-41.

Key Words: colorectal cancer, Lynch syndrome, HNPCC

\section{CLINICAL CONSIDERATIONS}

\section{Definitions}

- Lynch syndrome is defined as a hereditary predisposition to CRC and certain other malignancies (e.g., endometrial and gastric cancer) as a result of a germline mismatch repair (MMR) gene mutation. Lynch syndrome includes both individuals with an existing cancer and those who have not yet developed cancer.

- The associated MMR gene mutations are inherited in an autosomal dominant manner.

- Analytic validity refers to a test's ability to accurately and reliably measure the genetic characteristic (e.g., genotype, mutation, polymorphism) of interest. 
- Clinical validity defines how well test results correlate with the intermediate or final outcomes of interest. This is usually reported as a clinical sensitivity/specificity.

\section{Patient population under consideration}

These recommendations apply to all individuals with a new diagnosis of CRC. An estimated 2-4\% can be identified as having Lynch syndrome.

\section{Preliminary (screening) tests}

Microsatellite instability (MSI) testing or immunohistochemical (IHC) testing (with or without BRAF mutation testing) of the tumor tissue are examples of preliminary testing strategies that could be used to select patients for subsequent diagnostic testing. Diagnostic testing involves MMR gene mutation (and deletion/duplication) testing of the proband, usually using a blood sample. Lynch syndrome is most commonly caused by mutations in the two MMR genes MLH1 and MSH2; less commonly by mutations in MSH6 and PMS2.

Clinical performance (sensitivity/specificity) to identify Lynch syndrome:

- $80-91 \%$ sensitivity of MSI testing among those with MLH1 or MSH2 mutations, depending on MSI panel composition; associated specificity is $90 \%$.

- $55-77 \%$ sensitivity of MSI testing among those with MSH6 (or PMS2) mutations, depending on panel composition; associated specificity is $90 \%$.

- $83 \%$ sensitivity of IHC testing, regardless of MMR gene involved; associated specificity of $89 \%$.

- Virtually $100 \%$ of individuals with Lynch syndrome do not carry the $B R A F$ mutation, whereas $68 \%$ of those without Lynch syndrome do. BRAF mutation testing is usually restricted to CRC cases with absent staining for $M L H 1$.

- An estimated $84 \%$ of the individuals with Lynch syndrome can be identified with current methods for DNA sequencing and deletion analysis. The 16\% not detectable are associated with PMS2 mutations. Testing of this gene has only recently become commercially available, and its use was not included in this review. This rate is only achievable if all newly diagnosed CRC cases are tested.

\section{Treatment/follow-up of probands and relatives}

Evidence does not exist to make specific recommendations for changes in CRC treatment in probands. The EWG recommends that probands be informed of the advantages of contacting blood relatives to offer counseling and targeted testing to diagnose Lynch syndrome. Among relatives diagnosed with Lynch syndrome (MMR positive), more frequent colonoscopies are indicated and should begin at an earlier age than recommended for average risk individuals. Increased surveillance results in reduced rates of colon cancer and death from all causes. Among women with Lynch syndrome (both probands and relatives), additional surveillance for early identification of endometrial cancer may be considered, but there is less evidence to support it.

\section{Other considerations}

- The general debate on the issue of consent is acknowledged. However, because of the potential impact on the patient's relatives, the EWG recommends that individuals with newly diagnosed CRC should be routinely offered counseling and educational materials aimed at informing them and their relatives of the potential benefits and harms associated with genetic testing to identify Lynch syndrome.

- Protocols for sample collection, laboratory testing, and reporting of results need to be instituted, as well as for contacting, educating, testing, and following relatives with Lynch syndrome.

\section{Other approaches}

Family history is an important risk factor for CRC in the general population. Among individuals with newly diagnosed CRC, however, family history is less useful as the first step in identifying Lynch syndrome than strategies involving the analysis of tumor samples (e.g., MSI, IHC). The application of Amsterdam and Bethesda criteria has resulted in variable and generally poor performance in identifying Lynch syndrome. Therefore, the EWG does not recommend the use of family history to exclude individuals with newly diagnosed cancer from the offer of genetic testing.

\section{Economic considerations}

Costs per Lynch syndrome case detected depend on the testing strategy selected; higher costs are associated with higher sensitivity. Total program costs are highest when no preliminary tests are employed (e.g., all individuals with newly diagnosed $\mathrm{CRC}$ are offered DNA sequencing).

\section{BACKGROUND AND CLINICAL CONTEXT FOR THE RECOMMENDATION}

CRC is the second leading cause of cancer-related death in the United States in both men and women, with an estimated 52,000 deaths in 2007. ${ }^{1}$ About 1 in 30 CRC patients $(2-4 \%)$ have Lynch syndrome. ${ }^{2}$ When other relatives are found to carry a deleterious MMR gene mutation, they are also classified as having Lynch syndrome, because they are predisposed to developing these cancers, as well. The EWG avoids the term hereditary nonpolyposis colorectal cancer (HNPCC) because it now adds confusion to the understanding of this disorder. HNPCC has been applied to families meeting only limited family history criteria and to individuals with CRC having MSI-high test results, but no vertical transmission of a MMR gene mutation. ${ }^{3,4}$

The four MMR genes of major interest are $M L H 1, M S H 2$, MSH6, and PMS2. Mutations in MLH1 and MSH2 together account for the majority of Lynch syndrome cases diagnosed; mutations in MSH6 and PMS2 are less common. The risk of CRC in individuals with Lynch syndrome is high for both a second primary CRC in the patient (estimated at $16 \%$ within 10 years), and a new cancer in a first- or second-degree family member with Lynch syndrome (about $45 \%$ for men and 35\% for women by age 70). ${ }^{5}$ Changing management of both patients and relatives with the MMR gene mutation has the potential for reducing $\mathrm{CRC}$-related morbidity and mortality. To better understand the utility of DNA testing strategies in reducing morbidity and mortality from Lynch syndrome, EGAPP commissioned an evidence-based review to address an overarching question regarding the following specific clinical scenario:

Does risk assessment and MMR gene mutation testing in individuals with newly diagnosed CRC lead to improved outcomes for the patient or relatives, or is it useful in medical, personal, or public health decision making? 


\section{REVIEW OF SCIENTIFIC EVIDENCE}

This statement summarizes the supporting scientific evidence used by the EWG to make recommendations regarding the use of testing strategies to identify Lynch syndrome (presence of a MMR gene mutation) among newly diagnosed cases of CRC.

\section{Methods}

EGAPP is a project developed by the National Office of Public Health Genomics at the Centers for Disease Control and Prevention to support a rigorous, evidence-based process for evaluating genetic tests and other genomic applications that are in transition from research to clinical and public health practice in the United States ${ }^{6}$ A key goal of the EWG is to develop conclusions and recommendations regarding clinical genomic applications, and to establish clear linkage to the supporting scientific evidence. ${ }^{7}$ The EWG members are nonfederal multidisciplinary experts convened to establish methods and processes, set priorities for review topics, participate in technical expert panels for commissioned evidence reviews, and develop and publish recommendations.

EGAPP commissioned an evidence review through the Agency for Healthcare Research and Quality (AHRQ); the Tufts New England Medical Center Evidence-based Practice Center conducted the review. ${ }^{8}$ The review focused on the accuracy of diagnostic strategies for HNPCC, and the implications of testing to individuals with $\mathrm{CRC}$ and their families. It was anticipated that data might not be available to directly answer the overarching question. The EWG, therefore, constructed an analytic framework and key questions to address different components of evaluation (e.g., analytic and clinical validity, intermediate outcomes of interest, and clinical utility) for the purpose of providing relevant indirect evidence of efficacy. Established methods were followed in conducting this review. ${ }^{9}$ A Technical Expert Panel that included three EWG members was available to provide expert guidance during the course of the review. The final report, "Hereditary Nonpolyposis Colorectal Cancer: Accuracy of Diagnostic Strategies and Implications to Patients with Colorectal Cancer and Their Families," is available online. ${ }^{8}$

In addition, a technical contractor with experience in evidence review collaborated with EGAPP staff and consultants to conduct a supplementary targeted evidence review ${ }^{5}$ based on EGAPP methodology. ${ }^{10}$ This supplementary review was initiated because Lynch syndrome emerged as being of more specific interest than the less well-defined clinical constellation of HNPCC, and because EWG members requested additional information to address questions dealing with impact of testing strategies on relatives

EWG members reviewed the AHRQ evidence report, the supplementary targeted review, and key primary publications in detail, and examined other sources of information to address specific gaps in the evidence. The writers of the supplementary report and these EGAPP panel members further collaborated in constructing simple economic models to assist in analyzing the limited evidence available on clinical utility and in estimating how various testing strategies might function in practice. The final EGAPP recommendation statement regarding the use of testing strategies aimed at reducing morbidity and mortality from Lynch syndrome was formulated based on magnitude of effect, certainty of evidence, and consideration of contextual factors (e.g., severity of disorder, family considerations, and costs).

\section{Technology}

Strategies for risk assessment are defined in this review as a test or a specific series of tests offered to individuals with newly diagnosed CRC to identify those at sufficient risk for Lynch syndrome to be candidates for MMR gene testing. Based on the AHRQ evidence report, it was decided not to use the family history as an initial screening test (e.g., Amsterdam II or Bethesda criteria) because of the difficulty and costs of obtaining reliable family history and the overall poor sensitivity and specificity of this approach as a first step in identifying risk for Lynch syndrome in this clinical scenario. Possible preliminary tests include either MSI of tumor tissue that can identify the loss of MMR gene function, or IHC testing that identifies the absence of MMR gene protein in tumor tissue. Direct testing of the patient's DNA can then be performed by sequencing to identify deleterious mutations in MMR genes, and multiplex ligationdependent probe amplification (MLPA) to detect deletions in MMR gene exons. Testing for the BRAF V600E mutation is also being evaluated for use in patients whose IHC study indicates absence of the MLH1 protein. BRAF mutation testing is associated with methylation abnormalities of the $M L H 1$ promoter region which are not found in association with $M L H 1$ mutations. Individuals found with the $B R A F$ mutation are unlikely to have Lynch syndrome and, therefore, can avoid the need for expensive MMR gene testing.

\section{Analytic validity}

Analytic validity refers to a test's ability to accurately and reliably measure the analyte or genotype of interest, and includes measures of analytic sensitivity and specificity, assay robustness, and quality control. Three preliminary tests (MSI, IHC, and $B R A F$ ) are relevant for Lynch syndrome, as well as diagnostic testing for mutations in specific MMR genes via sequencing, and for MMR gene deletions by MLPA. Although a comprehensive review of these tests was not performed, general information regarding these tests is summarized below.

- DNA sequencing is considered a gold standard, but the actual analytic performance is difficult to estimate. A recently instituted European external proficiency testing program is focused on sequencing methodology rather than sequencing a specific gene. ${ }^{11}$ It is not yet known whether this approach will serve as an adequate measure of analytic validity.

- MSI testing is offered in many laboratories in the United States, and a high proportion will participate in the College of American Pathologists (CAP) external proficiency testing program (Molecular Pathology, MSI). ${ }^{12}$ Based on those program results, the analytic performance was high, but deficiencies were identified. Participant summary reports suggest that general adherence to best practices (e.g., documenting a high proportion of tumor cells, using three or more mononucleotide repeats) may be associated with higher analytic validity.

- IHC testing for MMR gene proteins is not currently subjected to CAP external proficiency testing, but IHC testing for other proteins (HER2, CD117, ER, CD-20, or EGFR) is offered as part of the CAP Immunohistochemistry Survey. ${ }^{13}$

- $B R A F$ mutation testing is less available than these other tests. Given that this test is aimed at identifying a single mutation, analytic validity is likely to be high; similar to that found through CAP proficiency testing for other single mutation tests such as the gene associated with 
hemochromatosis. ${ }^{14} B R A F$ mutation testing has very high clinical validity, as few reported MMR gene mutation carriers have also been found to carry this mutation; this provides indirect evidence of high analytic validity.

\section{Clinical validity}

The clinical validity of a genetic test defines how well test results correlate with the intermediate or final outcomes of interest. In this clinical scenario, the evidence for clinical validity is dispersed among studies examining MSI, IHC, BRAF and MMR gene testing, singly and in various combinations. MMR gene testing for one of the mutations of interest is the standard for defining Lynch syndrome. Thus, the EWG examined evidence comparing performance of MSI, IHC, and $B R A F$ as preliminary tests to identify individuals who should be offered diagnostic MMR gene testing.

\section{Microsatellite instability testing}

To determine clinical sensitivity of MSI testing, the ideal study would be to enroll individuals consecutively diagnosed with CRC from a typical population and perform MMR gene mutation testing on all, followed by MSI testing on those identified with Lynch syndrome. No such studies were found. Of 11 studies meeting inclusion criteria (examining a total of 150 patients with Lynch syndrome), only one was population based, but it was restricted to younger probands. ${ }^{5}$ The review was further complicated in that studies did not use the same markers (or the same number of markers) in the MSI panel, with some using as few as two and others as many as 11. A high proportion of mutations in the $M L H 1$ and $M S H 2$ genes can be associated with MSI-high results; about $89 \%$ if three or more mononucleotide markers are used. Sensitivity for MSH6 is probably lower, estimated at $77 \%$, even with a comprehensive panel. Current practice in clinical laboratories may result in lower performance than in research laboratories. Six studies provide information regarding clinical specificity, leading to an estimate of approximately $90.2 \%$ (false positive rate of $9.8 \%$ ). Two used only one mononucleotide marker (BAT26) to define MSI status and, as might be expected, both showed higher specificities (lower false positive rates) than the consensus.

\section{Immunohistochemical testing}

The optimal study design to determine clinical sensitivity of IHC testing would be similar to that for MSI; none were identified. Nine studies met inclusion criteria (examining a total of 149 patients with Lynch syndrome). ${ }^{5}$ Sensitivity for MLH1, MSH2, and MSH6 are each estimated at $83 \%$, based on seven studies for $M L H 1$ and $M S H 2$, and five studies for MSH6. Two studies were informative with respect to specificity, leading to an estimate of approximately $90 \%$ (false positive rate of $10 \%)$.

\section{BRAF V600E mutation testing}

About $90 \%$ of the mutations in the BRAF gene in CRC tumors are accounted for by a transversion (1799 T $>$ A), identified as V600E. The BRAF mutation is often present when the promoter region of the $M L H 1$ gene is methylated (methylation is the most common cause of absent MLH1 staining). When the BRAF V600E mutation is present, a deleterious MMR gene mutation has not yet been reported. These characteristics can be useful in determining which patients with absent MLH1 staining should be offered MLH1 gene sequencing. Among the three studies with useable results, no
$B R A F$ mutations were found among 42 Lynch syndrome patients with absent MLH1 staining, whereas $68 \%$ of sporadic cancers (e.g., MLH1 absent staining, but no detectable MMR gene mutation) had the $B R A F$ mutation. ${ }^{5}$ This reduces the number of patients needing MMR gene sequencing without reducing clinical sensitivity. Indirect evidence and gray data support this finding.

\section{Conclusions}

The EWG found convincing evidence that the sensitivity of MSI testing is about $89 \%$ for mutations in $M L H 1$ and $M S H 2$, with a lower sensitivity of about $77 \%$ for mutations in MSH6 (and PMS2). Sensitivity is higher when three or more mononucleotide markers are included in the panel. Specificity is estimated to be $90.2 \%$, with an adequate level of evidence. There is also convincing evidence that the sensitivity of IHC testing is $83 \%$, regardless of the underlying MMR gene mutation. Specificity is more variable, with a central estimate of $88.8 \%$ and an adequate level of evidence. Inadequate evidence is available to determine the distribution of mutations in the MMR genes, but preliminary estimates are 32\% MLH1, 39\% MSH2, 14\% MSH6, and $14 \% P M S 2$. Adequate evidence is available to estimate sensitivity (69\%) and specificity $(100 \%)$ for $B R A F$ mutation testing among newly diagnosed CRC cases with absent IHC staining for MLH1.

\section{Clinical utility}

The clinical utility of a genetic test is the likelihood that using the test to guide management will significantly improve health-related outcomes. In this clinical scenario, the question is whether a multistep testing strategy leads to improved clinical outcomes in patients or their relatives. The EWG examined a chain of evidence ${ }^{10}$ constructed from studies that individually assessed the components of clinical utility that might provide indirect evidence for clinical utility. At the highest level, these include whether testing leads to changes in clinical management for patients or relatives, and whether such changes in clinical management result in changes to outcomes, with attention to both benefits and harms. In each of these areas EGAPP found limited but promising evidence suggesting that testing can improve outcomes.

\section{Clinical management}

Evaluating clinical management involves answering the following two questions: (1) are management options for patients and relatives with an MMR mutation different from those without an MMR mutation; and (2) does knowledge of MMR mutation status change management decisions?

- Probands with Lynch syndrome-The EWG found a variety of surgical and medical management options for Lynch syndrome patients with $\mathrm{CRC}$, but was unable to identify any comparative studies. Subtotal colectomy with ileorectal anastomosis is recommended as a reasonable alternative to segmental resection in these cases, but it has not been shown to be superior at follow-up. No alteration in chemotherapy is currently recommended for Lynch syndrome patients, although a small body of evidence suggests that MSI-high tumors are relatively resistant to 5-fluorouracil and more sensitive to irinotecan. ${ }^{5}$ Further clinical trials will be necessary before clinical management recommendations are changed for Lynch syndrome patients with cancer.

- Family members with Lynch syndrome-Clinical management of first- and second-degree relatives of patients with 
Lynch syndrome begins with counseling and genetic testing and then includes increased surveillance for relatives found to have Lynch syndrome. Seven studies on the question of counseling and testing showed that about half of the relatives received counseling, and $95 \%$ of these chose MMR gene mutation testing. ${ }^{5}$ Among the seven studies that examined how testing affects surveillance among relatives with Lynch syndrome, uptake of colonoscopy was high beginning at age $20-25$ years, ranging from $53 \%$ to $100 \%$. Colonoscopy is recommended every $1-2$ years for both patients and their relatives with Lynch syndrome, beginning at age $20-25$ years. ${ }^{15}$ Colonoscopy risks include nausea, abdominal pain, dizziness, bleeding (2-21/1000 procedures), perforation ( $0.3-3.0 / 1000$ procedures), and death (0.0-0.2/1000 procedures).

- Risk-reducing colorectal resection in relatives is generally not recommended because of its inherent morbidity and rare mortality, but has been suggested as an option in special circumstances. No data are available regarding how often this option is presented and accepted. A decision analytic study ${ }^{16}$ suggested that subtotal colectomy in patients under age 47 with Lynch syndrome increased life expectancy by $1-2.3$ years. Indirect evidence from one study suggested that identification of MMR mutations was associated with better prognosis of CRC.

- Female probands and relatives with Lynch syndrome-In women with Lynch syndrome, transvaginal ultrasound and endometrial biopsy every 1-2 years, beginning at age 30-35 years, have been recommended by some, because of the associated risk for endometrial cancer. Two studies have shown that adherence to surveillance is higher in women found to carry a mutation. ${ }^{17,18}$ Hysterectomy and bilateral salpingo-oophorectomy is also an option, although not recommended. ${ }^{5}$

\section{Outcomes}

Using the chain of evidence methodology, ${ }^{10}$ the EWG found adequate evidence that an appropriate testing strategy could lead to acceptable changes in management that can improve clinical outcomes for patients and their relatives. Although there are no randomized trials exploring whether systematic colonic surveillance (e.g., colonoscopy) is effective in reducing Lynch syndrome-related morbidity and mortality, one long-term, nonrandomized controlled study from Finland followed 252 relatives at high risk of having Lynch syndrome. ${ }^{19}$ Mutation testing became available during the course of the study, and all colon cancers that developed were found in relatives who carried a mutation. Using an intention to treat analysis, 10 incident CRC cases $(8 \%)$ occurred among those having colonic surveillance, whereas 26 incident CRC cases (22\%) occurred among relatives without such surveillance. This represents a $62 \%$ reduction in risk for $\mathrm{CRC}$ and a significant reduction in CRC-associated mortality among relatives of Lynch syndrome cases. Supporting evidence was also available from a cohort study of 2788 individuals from 146 Lynch syndrome families in the Netherlands (reduction in standardized mortality ratio between subjects with $(n=897)$ or without $(n=1073)$ colonic surveillance $(6.5$ vs. 23.9; $P<0.001) .^{20}$

In a retrospective study, 61 of 315 women with MMR gene mutations selected risk reducing surgery for endometrial cancer. ${ }^{21}$ After approximately 10 years, no endometrial cancers or ovarian cancers developed in the women with surgery, whereas a third of women who did not have surgery developed endometrial cancer, and 5.5\% developed ovarian cancer.
Studies reporting psychosocial sequelae of mutation testing find that distress among mutation carriers is usually short term and that noncarriers experience significant relief. ${ }^{5}$ Very few data are available with respect to concern about employment and insurance.

\section{Preliminary cost descriptions}

Existing economic analyses that included relatives with Lynch syndrome were reviewed and found to be inadequate (e.g., variability in assumptions and initial values, no consideration of impact on relatives, no assessment of IHC as the primary screening test or refined testing strategies that involve $B R A F$ or methylation testing). ${ }^{22-25}$ The EWG commissioned a basic economic analysis comparing selected strategies of combining MSI, IHC, $B R A F$, and MMR gene mutation testing for the identification of Lynch syndrome among individuals with CRC and their relatives. ${ }^{5}$ Four selected sample testing strategies were included. The outcome of interest was the cost per Lynch syndrome case detected (proband, and proband and relatives), total program costs through identification of Lynch syndrome individuals, and the associated incremental costs. Although this cost consequences analysis did not allow the EWG to recommend a specific strategy, the results were used in context with the other findings to inform its recommendation.

\section{Research gaps}

Research gaps were identified in four areas. Further studies in these areas could contribute substantially to refining recommendations:

1. Analytical validity - The technology for MSI, IHC, and MMR mutation testing has changed significantly in the last few years and might be expected to continue to do so. Better quality data regarding analytical validity of testing and laboratory proficiency testing should be a high priority. More information on the analytic validity of tests used to refine these strategies (e.g., BRAF mutation testing, direct methylation testing) is needed.

2. Clinical validity - Several testing strategies are available, and some are in limited clinical use. Better quality studies comparing their clinical validity are needed in typical populations of individuals with CRC. For example, how would a strategy beginning with MSI testing only, followed by MMR mutation testing in positives, compare with strategies beginning with IHC only, or with both? How would the addition of $B R A F$ or methylation testing change the overall clinical sensitivity and specificity? Are there circumstances under which collection of accurate family history information and use of Bethesda guidelines in a testing strategy might be effective in reducing the number of cases for which sequencing is warranted?

3. Clinical utility - CRC is common enough that a direct study that begins with genetic testing and follows through clinical outcomes should be possible in a multicenter protocol. Alternatively, higher quality studies of the individual components of clinical utility (e.g., changes in management, uptake of management recommendations, and long-term clinical outcomes) could be undertaken.

4. Cost-effectiveness analyses (CEA) - There are few CEAs for Lynch syndrome and they are limited in scope. Few have included the impact on relatives, none have examined IHC as the primary initial test, and they vary widely in their assumptions and initial values. In addition, none 
have looked at refined testing strategies that involve $B R A F$ or methylation testing. CEA studies could form the basis to recommend a specific testing strategy to identify Lynch syndrome, and such an analysis is currently underway in partnership with EGAPP. ${ }^{26}$

\section{Recommendations of other groups}

National Comprehensive Cancer Network (NCCN), Clinical Practice Guidelines in Oncology ${ }^{\mathrm{TM}}$ v.2.2008. ${ }^{27}$

\section{Inherited colon cancer}

- "HNPCC is the most common form of a genetically determined colon cancer predisposition . . a accounting for $2-3 \%$ of all colorectal cancer cases. Surveillance has been shown to reduce the risk of colorectal cancer and may be of benefit in the early diagnosis of endometrial cancer."

\section{HNPCC molecular work-up and genetic testing}

- "Mixed strategy (MSI testing for all colorectal cancer patients followed by $\mathrm{MSH} 2$ and $\mathrm{MLH} 1$ testing of MSI-high tumors) has been shown as the most cost-effective approach for HNPCC screening. However, conclusive data are not yet available that establishes which test is the most cost-effective screening mechanism in HNPCC"

- "Genetic screening for MSI is cost effective for patients with newly diagnosed colon cancer as well as for the siblings and children of mutation carriers."

- "When a mutation is found in the family, it offers an opportunity to provide predictive testing for at-risk relatives. Predictive testing can save people a lot of unnecessary procedures. It is important to consider genetic testing for at-risk family members when the family mutation is known."

American Society of Clinical Oncologists (ASCO) 2006, ${ }^{30}$ Update of Recommendations for the Use of Tumor Markers in Gastrointestinal Cancer.

- "2006 recommendation for use of microsatellite instability to determine prognosis. Microsatellite instability (MSI) ascertained by polymerase chain reaction (PCR) is not recommended at this time to determine the prognosis of operable colorectal cancer nor to predict the effectiveness of FU (fluorouracil) adjuvant chemotherapy."

\section{Contextual issues}

Major contextual issues considered by the EWG included

- With limited benefit of genetic testing to the CRC patient, the EWG recommends that informed consent should be obtained before MSI or IHC testing.

- Results of several studies comparing psychosocial outcomes between MMR gene mutation carriers and noncarriers, and changes in outcomes over time, have provided no indication of adverse events relating to genetic testing. Furthermore, changes in distress seem to be short lived among mutation carriers, and there may be decreases in colon cancer worry, general anxiety, and depression among noncarriers who do not have Lynch syndrome. The EWG found no substantial evidence to show that identifying Lynch syndrome via routine genetic testing would lead to adverse psychosocial outcomes.

- Evidence shows relatively high levels of uptake for counseling among first-degree relatives contacted, subsequent MMR gene mutation testing, and adherence to increased surveillance among relatives found to have Lynch syndrome. The
EWG concludes that the level of participation among relatives is sufficient to justify the resources needed to implement routine genetic testing strategies.

\section{REFERENCES}

1. Cancer Facts \& Figures 2007. American Cancer Society. Available at: http:// www.cancer.org/downloads/STT/CAFF2007PWSecured.pdf. Accessed June 5, 2008 .

2. Hampel H, Frankel WL, Martin E, et al. Screening for the Lynch syndrome (hereditary nonpolyposis colorectal cancer). N Engl J Med 2005;352:18511860 .

3. Jass JR. Hereditary non-polyposis colorectal cancer: the rise and fall of a confusing term. World J Gastroenterol 2006;12:4943-4950.

4. Lindor NM, Rabe K, Petersen GM, et al. Lower cancer incidence in Amsterdam-I criteria families without mismatch repair deficiency: familial colorectal cancer type X. JAMA 2005;293:1979-1985.

5. Palomaki GE, McClain MR, Melillo S, Hampel HL, Thibodeau SN. EGAPP supplementary evidence review: DNA testing strategies aimed at reducing morbidity and mortality from Lynch syndrome. Genet Med 2009;11:42-65.

6. Centers for Disease Control: Evaluation of Genomic Applications in Practice and Prevention (EGAPP). Available at: http://www.cdc.gov/genomics/ gtesting/EGAPP/about.htm. Accessed June 5, 2008.

7. Evaluation of Genomic Applications in Practice and Prevention (EGAPP): Implementation and Evaluation of a Model Approach. Available at: http:// www.egappreviews. org/. Accessed June 5, 2008.

8. Hereditary nonpolyposis colorectal cancer: diagnostic strategies and their implications [structured abstract]. Rockville, MD: Agency for Healthcare Research and Quality, 2007. Available at: http://www.ahrq.gov/clinic/tp/ hnpcctp.htm. Accessed June 5, 2008.

9. Evidence-based practice centers overview. Rockville, MD: Agency for Healthcare Research and Quality, 2007. Available at: http://www.ahrq.gov/ clinic/epc/. Accessed June 5, 2008.

10. Teutsch SM, Bradley LA, Palomaki GE, et al. The Evaluation of Genomic Applications in Practice and Prevention (EGAPP) initiative: Methods of the EGAPP Working Group. Genet Med 2009;11:3-14.

11. Patton SJ, Wallace AJ, Elles R. Benchmark for evaluating the quality of DNA sequencing: proposal from an international external quality assessment scheme. Clin Chem 2006;52:728-736.

12. College of American Pathologists. Enhance your molecular pathology analyses. Available at: http://www.cap.org/apps/cap.portal?_nfpb=true\&cntvwrPtlt actionOverride $=\% 2$ Fportlets $\% 2$ F contentViewer $\% 2$ Fshow \& windowLabel $=$ cntvwrPtlt\&cntvwrPtlt \%7BactionForm.contentReference $\% 7 \mathrm{D}=$ news_service $\%$ $2 \mathrm{~F} 0501 \% 2 \mathrm{~F} 0501$ _MPA.html\&_state $=$ maximized \&_pageLabel $=$ cntvwr. Accessed June 5, 2008

13. College of American Pathologists. HER2 testing guidelines and resources. Available at: http:// www.cap.org/apps/cap.portal?_nfpb=true\&cntvwrPtlt_actionOverride= $\% 2$ Fportlets\%2 FcontentViewer\%2Fshow\&_windowLabel=cntvwrPtlt\&cntvwr Ptlt \%7BactionForm. contentReference $\% 7 \mathrm{D}=$ committees $\% 2 \mathrm{Fcancer} \% 2 \mathrm{Fher} 2$ index.html\&_state $=$ maximized\&_pageLabel $=$ cntvwr. Accessed June 5, 2008.

14. Palomaki GE, Haddow JE, Bradley LA, et al. Estimated analytic validity of HFE C282Y mutation testing in population screening: the potential value of confirmatory testing. Genet Med 2003;5:440-443.

15. Vasen HF, Moslein G, Alonso A, et al. Guidelines for the clinical management of Lynch syndrome (hereditary non-polyposis cancer). J Med Genet 2007;44:353-362.

16. de Vos tot Nederveen Cappel WH, Buskens E, van Duijvendijk P, et al. Decision analysis in the surgical treatment of colorectal cancer due to a mismatch repair gene defect. Gut 2003;52:1752-1755.

17. Collins V, Meiser B, Gaff C, St John DJ, Halliday J. Screening and preventive behaviors one year after predictive genetic testing for hereditary nonpolyposis colorectal carcinoma. Cancer 2005;104:273-281.

18. Wagner A, van Kessel I, Kriege MG, et al. Long term follow-up of HNPCC gene mutation carriers: compliance with screening and satisfaction with counseling and screening procedures. Fam Cancer 2005;4:295-300.

19. Jarvinen HJ, Aarnio M, Mustonen H, et al. Controlled 15-year trial on screening for colorectal cancer in families with hereditary nonpolyposis colorectal cancer. Gastroenterology 2000;118:829-834.

20. de Jong AE, Hendriks YM, Kleibeuker JH, et al. Decrease in mortality in Lynch syndrome families because of surveillance. Gastroenterology 2006; 130:665-671.

21. Schmeler KM, Lynch HT, Chen LM, et al. Prophylactic surgery to reduce the risk of gynecologic cancers in the Lynch syndrome. $N$ Engl $\mathrm{J}$ Med 2006;354:261-269.

22. Breheny N, Geelhoed E, Goldblatt J, Ee H, O’Leary P. Economic evaluation of the familial cancer programme in Western Australia: predictive genetic testing for familial adenomatous polyposis and hereditary non-polyposis colorectal carcinoma. Community Genet 2006;9:98-106.

23. Olsen KR, Bojesen SE, Gerdes AM, Lindorff-Larsen K, Bernstein IT. Cost-effectiveness of surveillance programs for families at high and mod- 
erate risk of hereditary non-polyposis colorectal cancer. Int J Technol Assess Health Care 2007;23:89-95.

24. Syngal S, Weeks JC, Schrag D, Garber JE, Kuntz KM. Benefits of colonoscopic surveillance and prophylactic colectomy in patients with hereditary nonpolyposis colorectal cancer mutations. Ann Intern Med 1998;129:787796.

25. Vasen HF, van Ballegooijen M, Buskens E, et al. A cost-effectiveness analysis of colorectal screening of hereditary nonpolyposis colorectal carcinoma gene carriers. Cancer 1998;82:1632-1637.

26. Mvundura M, Palomaki GE, McClain MR, Coates RJ, Grosse SD. Cost effectiveness of genetic testing for Lynch syndrome among newly diagnosed colorectal cancer patients and their first-degree relatives [abstract]. Presented at the 30th Annual Meeting of the Society for Medical Decision Making. October 19, 2008. Philadelphia, PA.

27. National Comprehensive Cancer Network (NCCN), Clinical Practice Guidelines in Oncology ${ }^{\mathrm{TM}}$ v. 2.2008. Available at: http://www.nccn.org/professionals/physician_gls/PDF/colorectal_screening.pdf. Accessed December 8, 2008.

28. 2006 update of ASCO recommendations for the use of tumor markers in gastrointestinal cancer. J Oncol Pract 2006;2:314-316. 